\title{
Trial and error versus errorless learning of functional skills in patients with acute stroke
}

Julie Mount

Thomas Jefferson University

Samuel R. Pierce

MossRehab Hospital and Institute for Physical Therapy Education

Janet Parker

MossRehab Hospital

Rebecca DiEgidio

MossRehab Hospital

Russell Woessner

MossRehab Hospital

Follow this and additional works at: https://jdc.jefferson.edu/ptfp

Part of the Physical Therapy Commons

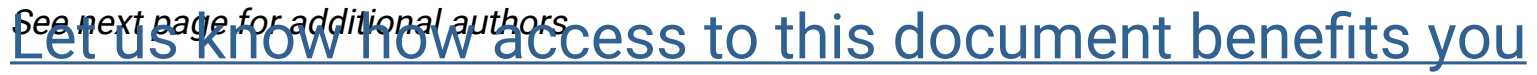

\section{Recommended Citation}

Mount, Julie; Pierce, Samuel R.; Parker, Janet; DiEgidio, Rebecca; Woessner, Russell; and Spiegel, Lenore, "Trial and error versus errorless learning of functional skills in patients with acute stroke" (2007). Department of Physical Therapy Faculty Papers. Paper 4.

https://jdc.jefferson.edu/ptfp/4

This Article is brought to you for free and open access by the Jefferson Digital Commons. The Jefferson Digital Commons is a service of Thomas Jefferson University's Center for Teaching and Learning (CTL). The Commons is a showcase for Jefferson books and journals, peer-reviewed scholarly publications, unique historical collections from the University archives, and teaching tools. The Jefferson Digital Commons allows researchers and interested readers anywhere in the world to learn about and keep up to date with Jefferson scholarship. This article has been accepted for inclusion in Department of Physical Therapy Faculty Papers by an authorized administrator of the Jefferson Digital Commons. For more information, please contact: JeffersonDigitalCommons@jefferson.edu. 


\section{Authors}

Julie Mount, Samuel R. Pierce, Janet Parker, Rebecca DiEgidio, Russell Woessner, and Lenore Spiegel 

Stroke

Julie Mount, PhD, PT ${ }^{\mathrm{a}}$, Samuel R. Pierce, PT, MS, NCS ${ }^{\mathrm{b}, \mathrm{c}}$, Janet Parker, MSPT, NCS ${ }^{\mathrm{b}}$, Rebecca DiEgidio, OTR/L ${ }^{b}$, Russell Woessner, PsyD ${ }^{b}$, Lenore Spiegel, $\mathrm{PhD}^{\mathrm{b}}$

5

\section{Abstract}

Objective: To compare the effectiveness of errorless learning versus trial and error learning for teaching activities of daily living to patients with acute stroke with or without explicit memory impairments. Design: Randomized crossover. Setting: Rehabilitation hospital. Participants: 33 adult subjects following an acute stroke. Intervention: Subjects were taught to prepare a wheelchair for a transfer and to put on a sock with a sock-donner. Tasks were taught using errorless learning or trial and error learning. Explicit memory was assessed using the Neurobehavioral Cognitive Status Exam. Main Outcome Measures: Days until subject was able to demonstrate retention of the task, and success or failure at carry-over to a similar task.

Results: No significant differences were found in days to retention for either functional task when taught using errorless learning or trial and error learning in subjects with or without explicit memory impairments. Carry-over was significantly better when trial and error learning was used for learning sock donning. Conclusions: When choosing the best learning method for patients undergoing rehabilitation for stroke, the nature of the task should be considered. Additional research is needed to identify the best approach for teaching activities of daily living and facilitating carry-over of learning in individuals with acute stroke.

Key words: Cerebrovascular Accident; Errorless Learning; Activities of Daily Living; Rehabilitation 
1 a. Department of Physical Therapy, College of Health Professions, Thomas Jefferson

2 University, Philadelphia, Pennsylvania

3 b. MossRehab Hospital, Philadelphia, Pennsylvania

4 c. Institute for Physical Therapy Education, Chester, Pennsylvania

5

6 Correspondence and reprint requests should be addressed to Julie Mount, PhD, Suite 830 Edison

7 Bldg., 130 South $9^{\text {th }}$ Street, Thomas Jefferson University, Philadelphia, PA 19130, phone\#:215

8503 6017, FAX \# 215503 3499, email: julie.mount@ jefferson.edu

9 Supported by a grant from the Albert Einstein Society. 
2 A major component of rehabilitation for individuals post acute stroke is teaching activities of

3 daily living (ADL). Motor skills such as walking, getting dressed, and wheelchair mobility are

4 often taught during the rehabilitation process in an attempt to maximize a patient's independence.

5 Two methods for teaching ADL's to patients post neurological injury are errorless learning (EL)

6 and trial and error learning (TEL). EL is a method that is structured so that the subject is

7 prevented from making errors while learning a task. TEL is a process in which the subject is

8 encouraged to try to guess or figure out the correct response and learn from any errors made.

9 Evidence suggests that TEL results in better retention of skills than EL for individuals without

10 memory deficits ${ }^{1-3}$. EL has been reported to be more effective than the TEL for teaching retention

11 of information in people with cognitive disorders such as mental retardation ${ }^{4}$, Alzheimer's

12 disease $^{5}$, schizophrenia ${ }^{6}$, and acquired brain damage ${ }^{7,8}$. A meta-analysis by Kessels and de Haan ${ }^{9}$

13 reported a statistically significant effect size for the effectiveness of EL in teaching retention of

14 skills in patients with amnesia. However, the assessment of the effectiveness of TEL and EL in

15 teaching skills to patients post acute stroke has not been investigated.

17 The mechanism of EL training is unknown but has been attributed to both the use of residual 18 explicit memory ${ }^{10}$ and implicit memory ${ }^{8}$. Explicit memory refers to memory that we consciously

19 recall, such as facts and events. Implicit memory refers to improvement in performance that

20 occurs without conscious or intentional recollection. Evans et al ${ }^{11}$ hypothesized that EL would be

21 optimal for tasks that can be learned implicitly. During the rehabilitation of patients with acute

22 stroke, therapists teach many functional tasks that are primarily motor skills, which may lend

23 themselves well to implicit memory and an EL approach. Orrell et al, for example, demonstrated 
1 that balancing tasks, which are commonly taught during rehabilitation, are learned implicitly even among individuals taught using discovery learning and focusing on explicit rules. ${ }^{12}$ Pohl and colleagues ${ }^{13}$ reported that implicit memory was preserved in people with chronic stroke as demonstrated by the learning of a perceptual-motor task. Boyd and Winstein ${ }^{14}$ found that explicit verbal feedback was detrimental for the learning of a motor sequence in people with chronic stroke, which may suggest that the verbal feedback provided by therapists during TEL may interfere with implicit memory. EL may be effective in patients with poor explicit memory, which is a common impairment in patients with acute stroke ${ }^{15}$, because this population may have preservation of implicit memory ${ }^{16}$. The identification of explicit memory deficits in patients post acute stroke may be critical in determining whether that patient will benefit from instruction using EL.

While the studies cited above suggest that EL is successful in teaching specific skills to people with decreased explicit memory, most of the literature reporting the value of EL does not address carry-over of learning to similar skills. Carry-over is defined as the effect of learning one skill on the subject's ability to perform a skill that is similar to but partially different from the learned skill. Carry-over is important in clinical practice because the activities learned in rehabilitation may not be identical to the activities that must be performed after discharge. Multiple investigations have suggested that TEL is superior compared to EL in obtaining carry-over in individuals without neurological impairments ${ }^{1-3}$. Gollin and Savoy ${ }^{17}$ found that children three to ten years of age who learned using EL made a greater number of errors than those who learned using TEL when carry-over to a new situation was tested. On the other hand, Glisky ${ }^{18}$ demonstrated carry-over of information that was learned through EL in a sample of adults with 
T\&E versus Errorless Learning

2

explicit memory impairments. A comparison of the effectiveness of EL and TEL in facilitating carry-over of skills in patients with acute stroke has not been investigated to date.

A second factor that limits the application of the research literature on EL and TEL to clinical practice is that the skills typically studied are not the activities of daily living frequently taught in acute stroke rehabilitation. For example, word lists are commonly taught in studies of $\mathrm{EL}^{7-8}$ while functional activities such as getting dressed have not been examined. In addition, only subjects with chronic neurological dysfunction have been studied in the research literature so that the effectiveness of EL and TEL in patients with acute stroke is unknown. Research is needed to compare the effectiveness of EL and TEL of tasks that are typically taught in the rehabilitation of individuals with acute stroke.

The purpose of this research was to compare the effectiveness of using EL versus TEL when teaching functional skills to patients with an acute stroke with or without explicit memory impairments. The following hypotheses were tested:

1. Individuals with an acute stroke with intact explicit memory require fewer instructional sessions to learn a functional task using TEL as compared to EL.

2. Individuals with an acute stroke with impaired explicit memory require fewer instructional sessions to learn a functional task using EL as compared to TEL.

3. Carry-over to a variation of the learned functional task is greater among individuals with or without impaired explicit memory who succeed in learning the functional task using TEL as compared to EL. 


\section{Methods}

Participants

Participants in the study were 33 individuals admitted to a large rehabilitation hospital with a diagnosis of acute stroke confirmed by computerized tomography, magnetic resonance imaging, or clinical examination. The mean age of participants was 63 years (range of 31 to $85, \mathrm{SD}=12$ ). There were 18 males and 15 females, 21 with a right stroke and 12 with a left stroke. The mean time post stroke onset to the start of study participation was 21 days (range $=6$ to $89, \mathrm{SD}=19$ days). Patients were excluded from the study if upon initial evaluation they demonstrated proper technique for wheelchair set up without instruction or if they were previously taught the sockdonning task. Also, patients were excluded if they did not have the physical or perceptual ability to complete either task with verbal instructions due to severe weakness, unilateral neglect, apraxia, or spatial deficits. In addition, subjects were excluded if aphasia was documented in the medical record, if verbal instructions in English were not understood due to English not being the primary language, or if a sock-donning task could not be attempted secondary to obesity or comorbid lower extremity amputation. If the patient had an expected length of stay in the hospital, which was less than one week from the time of screening, the subject was excluded from the study. Figure 1 demonstrates the results of the recruitment efforts for this study.

All subjects without suspected memory impairments signed a consent form approved by the facility's institutional review board and their family contact provided verbal and/or written consent for their participation. Subjects with suspected memory impairments signed a consent 
1 form approved by the facility's institutional review board in the presence of their approved family contact who also gave signed consent for participation.

The design was a randomized crossover design. The crossover design was selected so that subjects could serve as their own controls. This was important because of the heterogeneity within the population and the difficulty recruiting and running large samples within an acute rehabilitation setting. Each subject learned two tasks, one using the EL instructional method, and one using the TEL method. Subjects were randomly distributed to groups, which varied the type

11 of instruction for each task and the order of task instruction. The Neurobehavioral Cognitive

12 Status Exam (NCSE) ${ }^{19}$ was administered to subjects upon admission to the rehabilitation

13 hospital. The four-word memory component of the NCSE was used as a screening tool to identify

14 the degree of memory impairment of subjects in the study. This test was selected because it is a

15 time-efficient screening tool commonly used in the rehabilitation setting for the assessment of

16 explicit memory. Possible scores on the memory component of the NCSE range from 0 to 12.

17 Subjects with scores of 8 or lower were considered to have impaired memory based on guidelines 18 in the manual for the $\mathrm{NCSE}^{19}$.

20 Task Descriptions

22 The functional tasks used for instruction were preparing a wheelchair for a transfer and putting 23 on a sock with a sock-donner ${ }^{\mathrm{a}}$. These tasks were chosen because they are commonly taught to 
1 patients with acute stroke in the inpatient rehabilitation setting, are easily broken down into

2 discrete steps, can be performed even if one side of the body is completely paralyzed, and are

3 unlikely to have been learned prior to the stroke. Subjects who participated in the study were

4 asked to refrain from practicing either task during their regularly scheduled therapy sessions or

5 during their daily routine. Also, the subject's treatment team was informed of their participation

6 in the study and was asked not to teach either task. Prior to instruction in the wheelchair task,

7 patients were transferred into a standard wheelchair with bilateral brake extensions, a footplate,

8 and a button release seatbelt if the wheelchair that they used in the hospital did not have those

9 specifications. The wheelchair was positioned next to an exercise mat with the less involved side

10 adjacent to the mat. Prior to instruction in the sock-donning task, the patient's own sock was

11 removed from his or her less involved foot and the patient was issued a standard white tube sock

12 and the sock-donner. The steps of the wheelchair and sock-donning tasks are listed in Table 1.

14 Methods of Instruction

16 Detailed protocols were developed for each instructional method for each task, including

17 decision trees indicating how the instructor should respond depending on the subject's behavior.

18 For tasks taught using TEL, the subjects were permitted to make errors during the task sequence

19 but were provided with progressively more specific verbal cues to correct the errors. After the

20 first error was committed during a trial-and-error trial, the researcher told the subject an error was

21 made and asked the subject to try again to complete the step. After the second error on the same

22 step, the researcher provided a multiple-choice cue to the subject. After the third error, the

23 subject was given a directed cue to tell them what part of the body, sock donner, or wheelchair 
needed to be addressed to complete the step. If the subject was still unable to complete the step after the directed cue, the researcher provided hand-over-hand assistance with verbal cues to teach the subject the correct step of the sequence. If the subject made only a verbal response or did not attempt to complete the next step of the task after a fifteen second period, it was considered an error and the next cue was provided. This rule structure for cueing was followed until the subject completed all five steps of the task.

During EL training sessions, the subject was first instructed not to attempt to perform the next step of the task unless he was confident that he was correct. The subject was told that if he was uncertain, he should ask the instructing therapist to show him the correct step. The therapist would then provide hand-over-hand instruction with verbal cues to complete the step. If a subject started to make an error during the performance of a step, the therapist would as quickly as possible stop the subject from making the error and provide hand-over-hand instruction with

14 verbal cues to perform the step correctly. This rule structure was followed until the subject completed all five steps of the task.

16

17 Errors were classified as verbal errors, errors of no response, errors of action, or errors of 18 sequence. An error was coded as a verbal error when a subject provided only a verbal response of sequence was defined as a step that was performed out of sequence. 
T\&E versus Errorless Learning

Procedure for Learning Sessions

3 At the start of each testing session for each task, the subject was first asked to correctly complete the task without verbal instructions. When a subject was able to correctly complete a task on two

5 consecutive trials without any physical assistance or verbal cues and prior to any instruction on that day, the subject was considered to have achieved retention. If retention was not achieved

7 after seven days of training, training was discontinued for the subject. Training was limited to

8 only seven days due to limitations in subject length of stay and the availability of data collectors

9 for extended periods of time.

11 If the subject was unable to successfully complete the task for two consecutive trials, instruction

12 was provided to the subject in the form of verbal and physical guidance. The instructor physically

13 moved the subject's limbs through performance of the task while providing verbal instruction.

14 The subject was then asked to try to perform the task without verbal and physical guidance. It

15 was during the subject's attempt to reproduce the performance that differential instructions and

16 feedback were given according to whether the task was being taught using TEL or EL. This

17 sequence of teaching the entire task followed by the subject being asked to reproduce the task

18 occurred twice each day for each task. A five-minute break was provided for subjects in between

19 the wheelchair and sock donning tasks.

21 Carry-over Testing

23 The day after retention was achieved, the subject was tested on a carry-over task, which was 
1 similar to the original task. Carry-over of the wheelchair task was assessed using a wheelchair with a different type of brakes and seatbelt. Carry-over of the sock-donning task was assessed using a sock donner with a different kind of strap and degree of flexibility. During performance of the carry-over tasks, the number of correct steps completed and the number and nature of errors were recorded. No physical or verbal instructions were provided to subjects while completing the carry-over task. If all the steps were performed correctly in the correct order, the subject was considered to have successfully demonstrated carry-over.

Training and Reliability of Instructors

Patients were instructed in the wheelchair and sock donning tasks by physical and occupational therapists that were employed at the rehabilitation hospital or by physical therapy students from a locally affiliated graduate program. Study investigators trained the instructors/raters through the use of role-playing of patient scenarios with each rater individually. Instructors had to complete a competency test in which the investigators acted like different patients who had strokes.

Instructors were required to complete all protocols for instruction of both tasks using both methods, and were required to score the "patient" performances during the instruction, retention, and carry-over tasks without any errors.

After videotaping the instructional and testing sessions for the first 16 subjects, these videotapes were used to assess the reliability of the instructors. Sessions that were easy to see on videotape and that represented both tasks, each with both instructional methods, and included all the possible types of errors during the instruction and included retention and carry-over trials were 
dubbed onto videotape. Instructors viewed this videotape and independently scored the nature of errors (which determined what follow-up instruction was to be given when teaching the task), the number of steps correctly completed during the retention task, and success or failure in the carryover trials. Among instructors who had participated in data collection at least once per week at the time of reliability testing, the kappa values indicated excellent reliability for scoring the nature of errors $(\mathrm{k}=0.82)$, retention trials $(\mathrm{k}=1.0)$, and carry-over trials $(\mathrm{k}=1.0)$. For instructors who had participated in data collection less than once per week, kappa values were excellent for scoring of the nature of errors $(\mathrm{k}=0.70$ and 0.86$)$ and retention trials $(\mathrm{k}=1.0)$ and excellent to

9 moderate for carry-over trials $(\mathrm{k}=1.0$ and 0.50$)$.

Data Analysis

12

13 Survival analysis was used to compare the incident rate (IR) of retention for errorless versus trial-

14 and-error method in subjects with impaired memory and intact memory. The incidence rate (IR)

15 is the number of subjects who succeeded in learning the task expressed as a proportion of the

16 number of subject-days, which is the number of subjects times the number of days that subjects

17 participated in the study. A higher IR indicates a more effective method. Survival analysis was

18 selected in order to take into account subjects who did not achieve retention during the seven-day

19 testing period. An analysis that could not take these subjects into account would systematically

20 eliminate data from the least successful subjects and provide misleading results. The non-

21 parametric log-rank test for survival analysis was used due to a non-normal distribution of the 22 data. 
1 There were imbalances in proportions of males vs. females and right versus left stroke among the subgroups. While none of these variables had a significant effect by itself, the lack of

3 significance may have been due to the small sample sizes, and therefore logistic regression was used to adjust for these imbalances when analyzing both the retention and the carry-over data.

5 When adjusted vs. unadjusted results were compared, the adjustments made no difference in the trends seen in the data. A Wald test adjusted by logistical regression was used to compare the odds ratios for success during the carry-over task for both the sock and wheelchair skills for subjects trained with EL versus TEL and for subjects with intact versus impaired memory. Chisquare was used to analyze the types of errors committed during learning trials in order to see if there were differences between the tasks. SPSS 13 for Windows was used to do the statistical

11 analysis ${ }^{\mathrm{b}}$. Alpha was set at $<0.05$ for all analyses.

\section{Results}

The median number of days required to learn the wheelchair task was 2.5 days when using EL and 3 days when using TEL. The median number of days required to learn the sock-donning task was 3 days when using EL and 2 days when using TEL. The median memory score for all subjects on the Neurobehavioral Cognitive Status Examination was 9 with a range from 3 to 12. as having intact memory. subjects (30/33) were able to learn the wheelchair task. Table 2 presents the incidence rates (IR) 
1 of the errorless vs. trial-and-error method for subjects who had impaired vs. intact memory. No significant differences were found in the effectiveness of the two instructional methods for enabling patients with different levels of memory to achieve retention of these functional skills. However, Figure 2 illustrates differences in the trends in the effectiveness of the two learning methods for the two tasks across memory status. it (11/27). For the sock-donning task, the method of instruction significantly affected the likelihood of a subject successfully achieving carry-over $(\mathrm{OR}=19.92, \mathrm{p}=0.03,95 \% \mathrm{CI} 1.34$ to 296) with TEL significantly improving the odds of carry-over. The memory status of the subjects

11 (intact vs. impaired) did not significantly affect the likelihood of a subject successfully achieving 12 carry-over on the sock-donning task $(\mathrm{OR}=0.59, \mathrm{p}=0.62,95 \% \mathrm{CI} 0.07$ to 4.69$)$. The wheelchair carry-over task was successfully completed by $47 \%$ of subjects who attempted it (14/30). The method of instruction (EL vs. TEL) did not significantly affect the likelihood of a subject successfully achieving carry-over on the wheelchair task $(\mathrm{OR}=0.86, \mathrm{p}=0.89,95 \% \mathrm{CI} 0.12$ to 5.98). Subjects with intact memory tended to be more likely to achieve carry-over than subjects with impaired memory ( $\mathrm{OR}=0.21, \mathrm{p}=0.09,95 \%$ CI 0.03 to 1.30 ). See Figure 3 .

8

During retention trials, there was no significant difference between the wheelchair task and the sock-donning task in the proportion of verbal errors (17 errors and 15 errors respectively; ${ }_{(.05)} X^{2}$ $(1)=0.101 ; \mathrm{p}=0.750)$ or errors of sequence $\left(117\right.$ errors and 108 errors respectively; $(.05) X_{(1)}^{2}=$ $0.284 ; p=0.594)$ that occurred. However, there were significant differences between the wheelchair task and the sock-donning task in the proportion of errors of action (79 errors and 256 
1 errors respectively; $\left.{ }_{(.05)} X^{2}{ }_{(1)}=103.35 ; \mathrm{p}<0.001\right)$ and errors of no response $(184$ errors and 42 errors respectively; $\left.{ }_{(.05)} X^{2}{ }_{(1)}=86.73 ; \mathrm{p}<0.001\right)$.

\section{Discussion}

5

The purpose of this study was to compare the effectiveness of EL and TEL for teaching ADL's

7 during acute stroke rehabilitation to subjects with or without explicit memory impairments. To our knowledge, no studies have examined the effectiveness of EL and TEL in patients with acute stroke using functional tasks commonly taught in rehabilitation. The first two hypotheses of our investigation were that subjects with intact explicit memory would be able to learn a functional task faster using TEL while subjects with impaired explicit memory would be able to learn a task faster using EL. The present study did not find significant differences in the effectiveness of TEL compared to EL for teaching functional tasks to subjects with an acute stroke with intact or

14 impaired explicit memory. An unexpected finding was the apparent interaction effect between the tasks and the instructional method. Further research is needed to determine whether different

16 functional skills lend themselves better to different instructional methods.

18 The third hypothesis was that successful carry-over to a variation of the learned functional task would be greater among individuals who learned the functional task using a trial-and-error

20 approach as compared to an errorless approach. The present investigation found that subjects

21 who were taught sock-donning using TEL were significantly more likely to successfully

22 complete a carry-over task as compared to subjects trained using EL, when differences in explicit memory were controlled for using logistic regression. While improved carry-over of new skills 
1 after training with TEL compared to EL has been reported in subjects without neurological

2 dysfunction ${ }^{1-3}$, there has been little research addressing the issue of carry-over for populations

3 with memory impairment ${ }^{9}$. We believe that this is the first investigation to report a significant 4 advantage in carry-over in subjects with acute stroke learning a functional task using TEL rather 5 than EL.

$7 \quad$ No significant difference was found in carry-over of the wheelchair task for subjects who learned using TEL versus EL. For the wheelchair task, carry-over appeared more dependent on whether the subject had impaired memory than on the method of instruction (see figure 3), although the effect of memory impairment did not achieve significance $(\mathrm{p}=.09)$. Further research is needed to explore the relationships between method of instruction and memory ability for individuals with

12 acute stroke learning different types of tasks.

14 The patterns that emerged in both learning and carry-over for these subjects with acute stroke were different for the two tasks (see figures 2 and 3), although the nonparametric statistics

16 required for this study did not allow us to test for a significant interaction effect. In designing this

17 study, an attempt was made to select equivalent functional tasks taught in acute rehabilitation so

18 that each subject could use a different method for each task and serve as his or her own control.

19 Setting up for a wheelchair transfer and using a sock-donner were selected due to similarities in

20 the tasks with respect to the number of steps involved, the novelty of the task for subjects, and

21 the ability of the tasks to be performed in subjects with motor impairments. So what differences

22 between these two tasks might account for the different relationships between explicit memory

23 ability and optimal learning methods? 
2 The nature of the errors committed by subjects while learning the different tasks was analyzed

3 with the hope of gaining insight into whether there were in fact differences in how the two tasks were learned. It was found that when learning the wheelchair task, subjects were more likely not to attempt a response. However while learning sock-donning, subjects were more likely to commit errors of action. It may be that errors of action occurred more frequently during sock-

7 donning because perceptual judgment was required to perform some of the steps correctly, such

8 as judging how far to pull the sock down, and how to position the sock-donner on the floor.

9 Evans et al $^{11}$ suggested that EL helps people with memory impairments when the task they are

10 performing lends itself to the use of implicit memory. Perhaps the sock-donning task did not lend

11 itself as well to implicit memory, and that is why EL did not seem to benefit subjects with

12 impaired explicit memory as they tried to learn the sock-donning task. If the nature of the task is

13 an important factor determining which learning method will be more effective in achieving

14 retention and carry-over, then additional research is required to identify what features of tasks are

15 critical to know when choosing the optimal learning method.

17 One of the strengths of this study is its external validity for health professionals working in

18 rehabilitation settings. Kessels and deHaan ${ }^{9}$ conducted a meta-analysis concluding that errorless

19 learning is effective for amnesic patients, however, they identified as a limitation of the meta-

20 analysis that few studies looked at the effectiveness of errorless learning in "real-world" settings.

21 Many of the studies included in the meta-analysis used artificial tasks such as word lists or

22 recognition of pictures of faces. Our study used tasks that are actually taught in acute

23 rehabilitation and these tasks were taught to patients during their acute rehabilitation stay. We 
narrowed the population to people with stroke, and consequently, our subjects had less severe deficits in explicit memory than the subjects typically examined in studies of EL. While Tailby and Haslam ${ }^{20}$ found that EL was superior to TEL for training word lists in subjects with various degrees of memory impairment, many EL studies involve only patients with severe explicit memory deficits and its effectiveness with less severely impaired individuals is less established.

Testing the effectiveness of EL versus TEL in a natural acute rehabilitation setting was a strength with respect to external validity, but it resulted in a number of limitations. Because our subjects were enrolled during their acute rehabilitation stage, results may have been affected by improvement in our subjects' cognitive status over the course of training due to rehabilitation and natural recovery occurring in individuals with acute stroke. In addition, by studying subjects undergoing acute stroke rehabilitation, our investigation was limited in the duration of training sessions due to subject fatigue and time commitments due to therapy. Patients were only available to participate in the research between four and five PM, after a full day of rehabilitation. Patients and staff are very busy during acute rehabilitation, and a number of subjects were lost because there was not time to complete their NCSE testing or because subjects found they were too tired to participate and they dropped out. Subjects were also lost due to early discharge for medical complications. The limit of seven days of training for each subject required due to anticipated hospital length of stay limited our ability to measure potential learning that could have occurred with more than seven days of training. While these factors were limitations in determining the efficacy of EL versus TEL, they represent the reality that patients experience when learning skills during acute rehabilitation. 
4

\section{Conclusion}

The present investigation suggests that for individuals with acute stroke, the effectiveness of EL compared to TEL may be dependent on the nature of the task to be learned. The TEL approach significantly improved carry-over of learning of the sock-donning task compared to EL, while there was no difference in carry-over of the wheelchair task between learning methods. Additional research is needed to identify the best approach for teaching different activities of daily living and facilitating carry-over of learning for individuals with acute stroke.

\section{Acknowledgments}

We would like to thank the subjects of this investigation who were generous with their time while undergoing acute inpatient stroke rehabilitation. Also, we would like to acknowledge the physical and occupational therapists from MossRehab and the physical therapy students from Thomas Jefferson University who participated as data collectors throughout the study.

\section{References}

[1] D.C.Prather, Trial and error learning versus errorless learning: training, transfer, and stress, Am J Psych 84 (1971), 377-86.

[2] R. Singer and L. Gaines, Effect of prompted and trial and error learning on transfer performance of a serial motor task, Am Ed Res J 12 (1975), 395-404.

[3] R.N. Singer and D. Pease, A comparison of discovery learning and guided instructional strategies on motor skill learning, retention, and transfer, Res Quart 47 (1976), 788-796. 
1 [4] P.G. Walsh, Teaching colour discrimination to the mentally handicapped, Irish J Psychol 7

2 (1985), 36-49.

3 [5] L. Clare, B.A. Wilson, G. Carter, K. Breen, A. Gosses, and J.R. Hodges, Intervening with 4 everyday memory problems in dementia of Alzheimer type: An errorless learning approach, $J$

$5 \quad$ Clin \& Exp Neuropsychology 22 (2000),132-146.

6 [6] R.E. O'Carroll, H.H.Russell, S.M. Lawrie, and E.C. Johnstone. Errorless learning and the 7 cognitive rehabilitation of memory impaired schizophrenic patients, Psycholog Med 29 (1999), 105-112.

[7] A. Baddeley, and B.A. Wilson, When implicit learning fails: amnesia and the problem of error elimination, Neuropsychologia 32 (1994), 53-68.

[8] B.A.Wilson, A. Baddeley, and J. Evans, Errorless learning in the rehabilitation of memory impaired people, Neuropsych Rehab 4 (1994), 307-326.

[9] R.P.C. Kessels, and E.H.F. de Haan, Implicit learning in memory rehabilitation: a metaanalysis on errorless learning and vanishing cues methods, J Clin Exp Neuropsychol 25 (2003), $805-14$.

[10] N.M. Hunkin, E.J. Squires, A.J. Parkin, and J.A. Tidy, Are the benefits of errorless learning dependent on implicit memory? Neuropsychologia 36 (1998), 25-36.

[11] J.J. Evans, B.A. Wilson, U. Schuri, J. Andrade, A. Baddeley, and O. Bruna O, T. Canavan, S. Della Sala, R. Green, R. Laaksonen, L. Lorenzi, and I. Taussik, A comparison or "errorless" and "trial-and-error" learning methods for teaching individuals with acquired memory deficits. Neuropsych Rehab 10 (2000), 67-101.

[12] A.J. Orrell, F.F. Eves, and R.S.W. Masters, Implicit motor learning of a balancing task, Gait and Posture. 23 (2006), 9-16. 
[13] P.S. Pohl, J.M. McDowd, D.L. Filion, L.G. Richards, and W. Stiers, Implicit learning of a perceptual-motor skill after stroke, Phys Ther. 81 (2001),1780-9.

[14] L.A. Boyd and C.J. Winstein, Impact of explicit information on implicit motor-sequence learning following middle cerebral artery stroke, Phys Ther. 83 (2003), 976-89.

[15] T. Pohjasvaara, T. Erkinjuntti, R. Vataja and M. Kaste M, Dementia three months after stroke, Stroke 28 (1997), 785-792.

[16] K.J. Sullivan and C.J. Winstein, Actions speak louder than words: a case study demonstrating declarative but not procedural memory deficits, Neurology Report. 21 (1997),49. [17] E.S. Gollin and P. Savoy, Fading procedures and conditional discrimination in children, $J$ Exp Analysis Beh 11 (1968), 43-51.

[18] E.L.Glisky, Acquisition and transfer of declarative and procedural knowledge by memoryimpaired patients: a computer data-entry task, Neuropsychologia 30 (1992), 899-910.

[19] The Northern California Neurobehavioral Group, Cognistat: The Neurobehavioral Cognitive Status Examination. Fairfax, CA.: The Northern California Neurobehavioral Group. 1995. [20] R. Tailby and C. Haslam, An investigation of errorless learning in memory-impaired patients: improving the technique and clarifying theory, Neuropsychologia 41 (2003),1230-40. Chicago, Illinois, 60606. 


\begin{tabular}{cll}
\hline Step Number & Wheelchair Task & Sock-Donning Task \\
\hline 1 & $\begin{array}{l}\text { Lock brakes on stronger side } \\
\text { Lock brakes on weaker side }\end{array}$ & $\begin{array}{l}\text { Place sock-donner between legs } \\
\text { Pull sock just to edge of sock-donner } \\
\text { with toe of sock tight }\end{array}$ \\
& Remove weaker foot from & $\begin{array}{l}\text { Use straps to lower sock-donner to } \\
\text { floor with open side facing up } \\
\text { footplate }\end{array}$ \\
4 & Lift up footplate & $\begin{array}{l}\text { Slip foot into sock-donner and pull } \\
\text { straps until the sock is on foot } \\
\text { Using the straps, remove the sock- } \\
\text { donner from the foot }\end{array}$ \\
\hline
\end{tabular}

3 Table 1: Steps of the wheelchair and sock-donning tasks. 


\begin{tabular}{|l|l|r|r|r|r|r|}
\hline Task & Impaired & \multicolumn{2}{|c|}{ Errorless } & \multicolumn{2}{|c|}{ Trial \& Error } & \\
\cline { 3 - 7 } & or Intact & $\mathbf{N}$ & \multicolumn{1}{|c|}{ IR } & N & \multicolumn{1}{c|}{ IR } & \multicolumn{1}{c|}{ P value } \\
\hline Wheelchair & Impaired & 6 & .462 & 8 & .207 & .21 \\
\cline { 2 - 7 } Preparation & Intact & 10 & .250 & 9 & .429 & .11 \\
\hline Sock- & Impaired & 8 & .259 & 6 & .333 & .39 \\
\cline { 2 - 7 } Donning & Intact & 9 & .318 & 10 & .267 & .74 \\
\hline
\end{tabular}

2

3 Table 2: Incidence Rates (IR), numbers of subjects in subgroups (N), and p values when

4 effectiveness of EL is compared to effectiveness of TEL for each task for subjects with impaired 5 and intact memory. 
T\&E versus Errorless Learning

\section{$1 \quad$ Figure Captions}

2 Figure 1: Study recruitment.

3 Figure 2: Effectiveness of the two learning methods for different levels of memory for the two

4 different tasks.

5 Figure 3: Percentages of subjectc who achieved carrvever for the two learning methods for

6 different levels of memory for th Assessed for eligibility $(\mathrm{n}=550)$

Excluded $(\mathrm{n}=503)$

Aphasia $(\mathrm{n}=151)$

Length of stay less than one week from screen $(\mathrm{n}=115)$

Diagnosis not stroke $(\mathrm{n}=59)$

Non-English speaking $(\mathrm{n}=29)$

Dementia $(\mathrm{n}=27)$

Declined participation $(\mathrm{n}=25)$

Independent with wheelchair task $(\mathrm{n}=25)$

Decreased arousal $(\mathrm{n}=25)$

Other $(\mathrm{n}=47)$
Drop out $(n=6)$ Unavailable for NCSE testing $(\mathrm{n}=2)$

Refusal (n=2) Medical discharge $(\mathrm{n}=1)$

Determined to be ineligible $(n=1)$
WC-trial and error;

Sock-errorless $(n=22)$
Consented to participate $(n=47)$

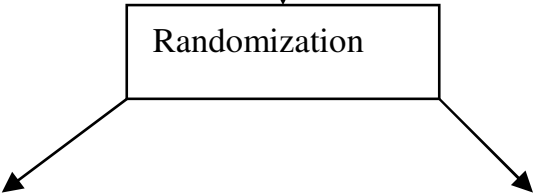

WC-errorless;

Sock-trial and error $(n=25)$
Participated

$(n=16)$
Participated $(\mathrm{n}=17)$
Drop out $(\mathrm{n}=8)$ Unavailable for NCSE testing $(\mathrm{n}=4)$

Medical discharge $(\mathrm{n}=2)$

Determined to be ineligible $(\mathrm{n}=2)$ 


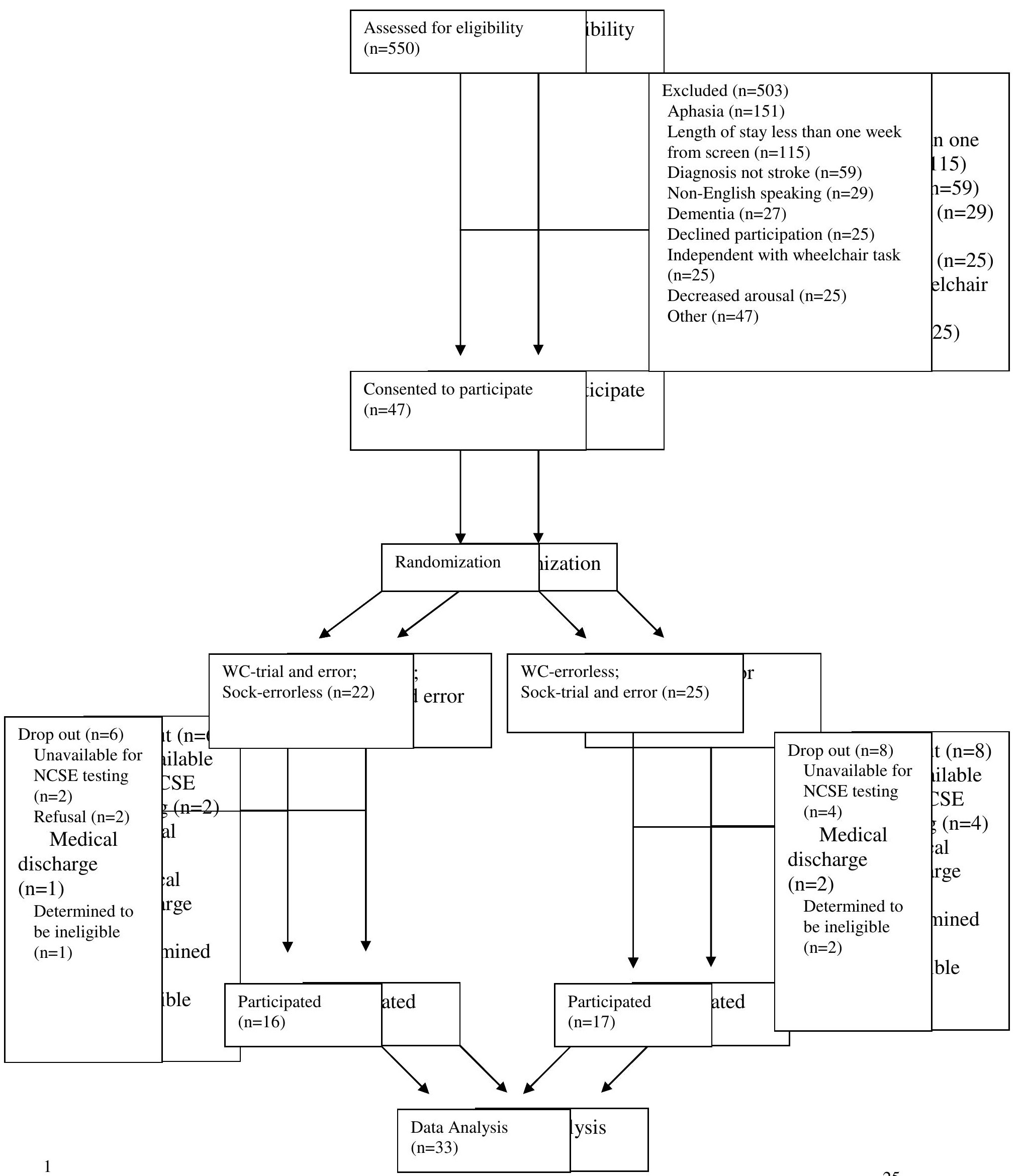


T\&E versus Errorless Learning

1 


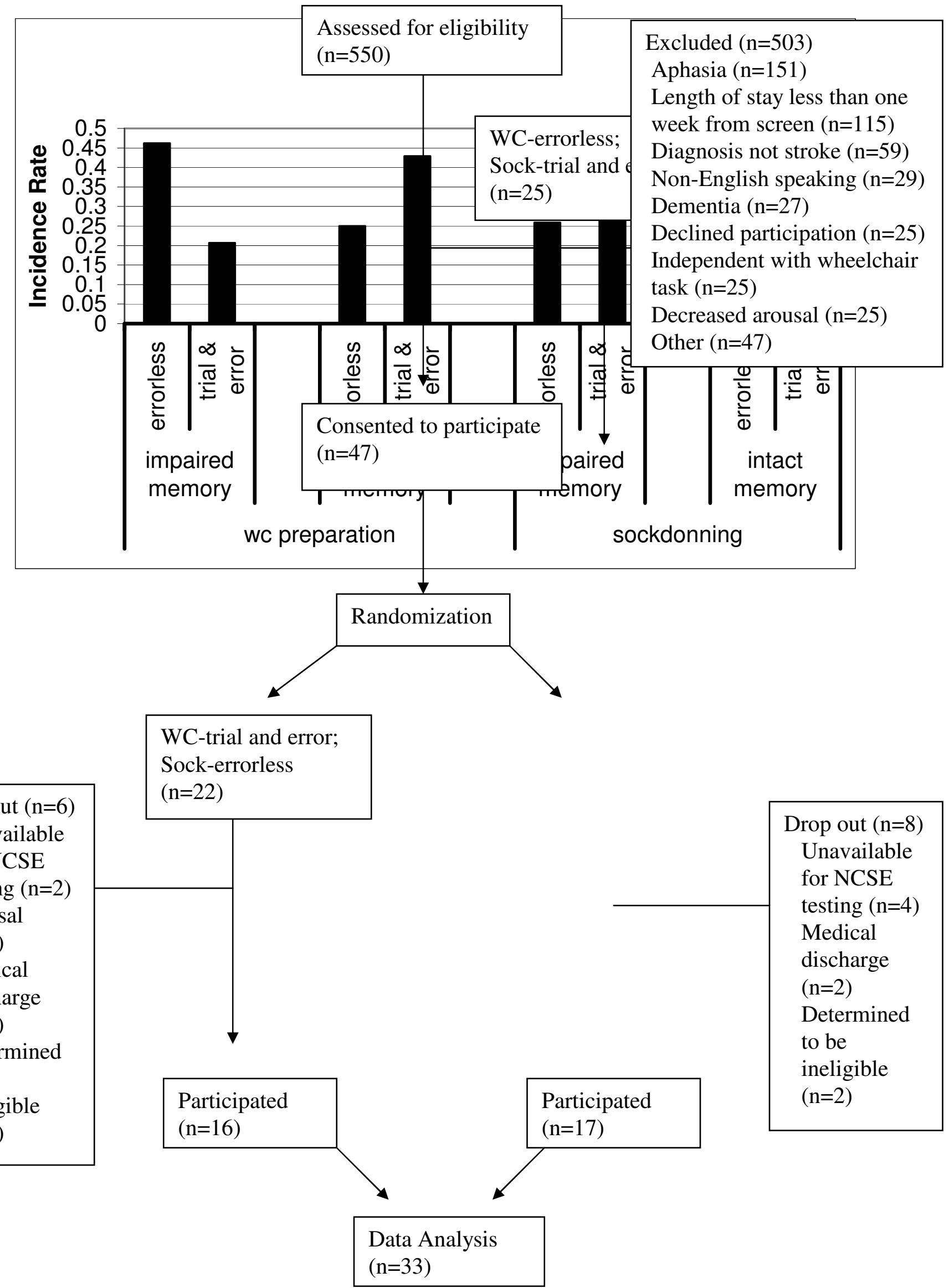




\section{Carryover}

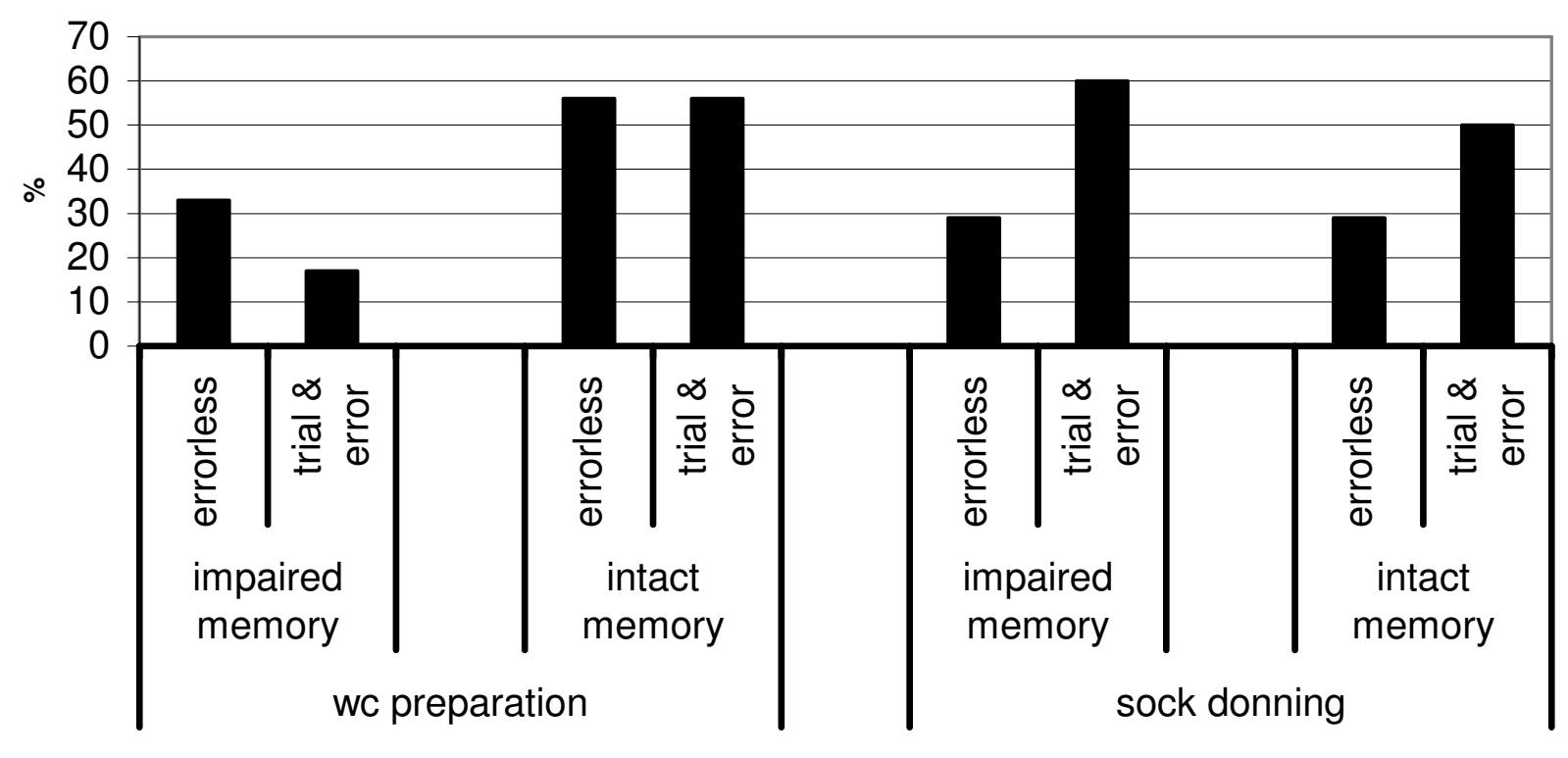


T\&E versus Errorless Learning

1 